\title{
Positive Sentinel Lymph Nodes are a Negative Prognostic Factor for Survival in T1-2 Oral/Oropharyngeal Cancer: A Long-Term Study on 103 Patients
}

\author{
Kavita M. Pattani ${ }^{1}$ and Joseph Califano ${ }^{1,2}$ \\ ${ }^{1}$ Department of Otolaryngology-Head and Neck Surgery, Johns Hopkins Medical Institutions, 601 N. Caroline Street, 6th \\ Floor, Baltimore, MD 21287-0910, USA; ${ }^{2}$ Johns Hopkins Head and Neck Surgery at GBMC, 6569 North Charles Street, \\ Physicians Pavilion West, Suite 200, Baltimore, MD 21204, USA
}

Detection and treatment of occult nodal metastasis and management of the clinically N0 neck have posed a challenge in head and neck squamous cell carcinoma (HNSCC). In an attempt to translate successful staging strategies employed in breast carcinoma and melanoma, sentinel node biopsy (SNB) has been investigated in the context of HNSCC over the past decade. The concept of sentinel node biopsy relies on the theory that the firstechelon node/s (sentinel node) will harbor the lymphatic flow from the tumor, and pathologic evaluation of this node will accurately reflect the nodal staging. ${ }^{1-3}$ In particular, early-stage oral-cavity and oropharyngeal cancers have been targeted for studies evaluating the utility of sentinel node biopsy, and the incidence of occult metastatic disease to the cervical lymph nodes is approximately $20-30 \%$ in low-stage oral-cavity cancers. ${ }^{4-6}$ Most investigations have focused on the possibility that accurate SNB may facilitate avoidance of staging neck dissections, particularly in patients with low-stage T1-2 oral-cavity cancer that would avoid postoperative radiotherapy with low-risk primary tumor and pathologically negative neck.

In this issue, Kovacs et al. address the prognostic value of sentinel lymph node biopsy in oral/oropharyngeal squamous cell carcinoma. With a moderate sample size of 103 patients, they reported no false negatives, and poor prognostic associations with patients that had a positive sentinel node. Although encouraging, there are several unique characteristics of this study that make it difficult to extrapolate these findings to routine clinical practice: (1)

(C) Society of Surgical Oncology 2008

First Received: 5 September 2008;

Published Online: 19 November 2008

J. Califano

e-mail: jcalifa@jhmi.edu the use of intra-arterial high-dose chemotherapy given 3-4 weeks prior to surgery for primary cancer is not widely adopted, and may have variable effects on clinical disease outcomes, lymphatic drainage patterns, or impacted micrometastatic spread to draining lymph nodes, (2) uniform use of fluorodeoxyglucose positron emission tomography (FDG-PET) may have stage-shifted patients such that the study population was enriched for pathologic N0 neck disease, and resulted in the low upstaging rate reported by these authors as compared with other published data. ${ }^{7-10}$

The prognostic significance of a positive sentinel node reported in this study is difficult to assess in a larger context as well because: (1) planned postoperative radiation was employed for patients with a T2 primary tumor with an N0 neck, a practice not uniformly applied, (2) only 1/9 patients who were upstaged by positive SNB actually received postoperative radiation, a usual standard of care, and (3) the reported diameter of all the positive nodes was $\geq 10 \mathrm{~mm}$ and the diameter of the metastasis averaged $4.6 \mathrm{~mm}$; it is unclear why these nodes did not fulfill computed tomography (CT) size criteria $(1.0 \mathrm{~cm})$ that designate a pathologic node.

A recent large meta-analysis in HNSCC conducted by Paleri et al. estimated a detection rate of $90 \%$ and a falsenegative rate of $10 \%$ for SNB. ${ }^{11}$ Although a false-negative rate of zero is reported by Kovacs et al., there were two patients who developed contralateral neck metastasis and one patient with submandibular gland cancer of unreported histology, reflecting a lack of consensus regarding the definition of a false-negative SNB in HNSCC. Of note, in two recent series evaluating SNB it was demonstrated that unanticipated contralateral sentinel lymph nodes can occur in $15-20 \%$ of patients. ${ }^{12,13}$ 
Continued studies regarding the utility of SNB may help in defining these issues. In particular, prospective randomized trials may help in determining clinical outcomes comparisons, as well as cost-benefit analyses regarding the use of SNB compared with staging neck dissection in appropriate patient cohorts.

\section{REFERENCES}

1. Morton DL, et al. Technical details of intraoperative lymphatic mapping for early stage melanoma. Arch Surg. 1992;127(4): 3929.

2. Morton DL, et al. Validation of the accuracy of intraoperative lymphatic mapping and sentinel lymphadenectomy for earlystage melanoma: a multicenter trial. Multicenter Selective Lymphadenectomy Trial Group. Ann Surg. 1999;230(4):453-63; discussion 463-5.

3. Alex JC, Krag DN. The gamma-probe-guided resection of radiolabeled primary lymph nodes. Surg Oncol Clin North Am. 1996;5(1): 33-41.

4. Pfister DG, et al. NCCN Practice Guidelines for Head and Neck Cancers. Oncology. 2000;14(11A) 163-94.

5. Pillsbury HC III, Clark M. A rationale for therapy of the N0 neck. Laryngoscope. 1997;107(10):1294-315.
6. Shah JP, Andersen PE. The impact of patterns of nodal metastasis on modifications of neck dissection. Ann Surg Oncol. 1994;1(6): 521-32.

7. Wagner A, et al. Validity of sentinel lymph node (SLN) detection following adjuvant radiochemotherapy (RCT) in head and neck squamous cell carcinoma (HNSCC). Technol Cancer Res Treat. 2007;6(6):655-60.

8. Kovacs AF, et al. Pattern of drainage in sentinel lymph nodes after intra-arterial chemotherapy for oral and oropharyngeal cancer. J Oral Maxillofac Surg. 2005;63(2):185-90.

9. Ross G. Rationale for sentinel node biopsy to stage NO head and neck squamous-cell carcinoma. Cancer Biother Radiopharm. 2004;19(3):273-84.

10. Stoeckli SJ, et al. The second international conference on sentinel node biopsy in mucosal head and neck cancer. Ann Surg Oncol. 2005;12(11):919-24.

11. Paleri V, et al. Sentinel node biopsy in squamous cell cancer of the oral cavity and oral pharynx: a diagnostic meta-analysis. Head Neck. 2005;27(9):739-47.

12. Carlson GW, et al. Management of malignant melanoma of the head and neck using dynamic lymphoscintigraphy and gamma probe-guided sentinel lymph node biopsy. Arch Otolaryngol Head Neck Surg. 2000;126(3):433-7.

13. Wagner JD, et al. Cervical sentinel lymph node biopsy for melanomas of the head and neck and upper thorax. Arch Otolaryngol Head Neck Surg. 2000.;126(3):313-21. 